\title{
Viscoelastic Properties of Starch and Non-Starch Thickeners in Simple Mixtures or Model Food
}

\author{
Ioanna G. Mandala
}

Additional information is available at the end of the chapter

http://dx.doi.org/10.5772/50221

\section{Introduction}

Design of new food products is one of the most important tasks in the food industry. Improving or controlling texture of foodstuffs leads to products with advanced functionalities e.g. creation of nursing-care foods, "ready-to swallow foods", soft or hard gels etc. (Funami, 2011).

Texture change can be achieved by adding hydrocolloids that in small quantities bind large amounts of water and can then control both structure and texture. Starches belong to the same category of hydrocolloids, although they are used in a wide range of products either as raw materials or as food additives. Starches can differ with respect to the amylose content depending on their origin, or can be structurally modified. Native starches could have negative aspects such as gel syneresis, retrogradation, breakdown, cohesive, rubbery pastes and undesirable gels formation (Whistler \& BeMiller, 1997), but this is not the case with modified starches. Moreover, modified food starches are less expensive and are more widely available than gums or other food stabilizers. A way to overcome shortcomings of native starches is their blending with polysaccharide hydrocolloids. Native or modified starches, and non-starch hydrocolloids are increasingly important ingredients in the modern health-conscious food industry (Techawipharat et al., 2008), considering that specific starch types such as resistant starch can be considered insoluble fibers as well.

This chapter aims at highlighting recent research in the field of viscoelastic properties of starches and their mixtures with some selected hydrocolloids. Furthermore, these interactions will be linked to the final rheological characteristics of specific products aimed at successful product development.

The control of texture in real foods with several ingredients can be achieved through viscoelasticity measurements of carbohydrate mixtures at low concentrations. This research 
can result in new products development that aims at specific texture characteristics. Examples are focused on oscillatory experiments and in some case correlations to viscous properties are presented. The role of rheology in current research is further shown and discussed through these examples.

\section{Oscillatory rheological method and hydrocolloids behavior}

Among different techniques used to distinguish between the solid and liquid-like characteristics of a food colloid, the best technique is to use an oscillatory rheological method (Dickinson, 1992). In an oscillatory rheological experiment, both stress and strain commonly present a sinusoidal variation. This is the most popular method to characterize viscoelasticity, since relative contributions of viscous and elastic response of materials can be measured. The cycle time, or frequency of oscillation, defines the timescale of these tests. By observing material response as a function of frequency, material can be tested at different timescales. The observation of material response at different frequencies is also referred to as mechanical spectroscopy (Stanley et al., 1996). Linear viscoelasticity is known as the region where stress and strain waves are set at such low values that stress is proportional to strain. This type of tests is also known as small amplitude oscillatory shear (SAOS). The relationship between stress and strain is then described and storage, loss modulus, complex shear modulus as well as dynamic viscosity can be measured. The storage dynamic modulus $\left(G^{\prime}\right)$ is a measure of the energy stored in the material and recovered from it per cycle while the loss modulus $\left(\mathrm{G}^{\prime \prime}\right)$ is a measure of the energy dissipated or lost per cycle of sinusoidal deformation (Ferry, 1980, Stanley et al., 1996 ). The ratio of the energy lost to the energy stored for each cycle can be defined by tano.

The viscoelastic behavior of a simple or more complex structure can be determined in the above way. Furthermore, as structure is not disrupted, changes including sol-gel transition, gel curing, aggregation, flocs creation etc. can be monitored. Generally speaking, rheological properties could be of high interest in a) product quality characterization b) process design and flow conditions analysis (e.g. pump sizing, filtration, extrusion etc.) c) design of new foodstuffs d) basic research on ingredients' interactions.

According to Roos-Murphy (1984) solutions and gels belong to the categories of entanglement solutions, weak gels and strong gels. Hydrocolloids including starch can belong to all of the three categories revealing the wide spectrum of structures they can adopt according to their own natural state and the environmental conditions found.

Entanglement solutions (e.g. guar gum solutions) present a strong dependence of both storage and loss modulus on frequency. Weak gels behavior (e.g. xanthan gum) is characterized by gel-type mechanical spectrum, whereas strong gels (e.g. amylopectin, amylose gels) present high storage modulus values irrespective of frequency, as junction zones among macromolecules are stable on a relatively long time scale. The spectrum of hydrocolloids can vary at different concentrations.

When at low frequency the loss modulus $G^{\prime \prime}$ is higher than the storage modulus $G^{\prime}$, both parameters vary sharply with frequency: $G^{\prime \prime}(\omega)$ and $G^{\prime}\left(\omega^{2}\right)$. This behavior is said to be 
typical of a liquid-like material. As frequency increases, $G^{\prime}(\omega)$ crosses $G^{\prime \prime}(\omega)$, the response of the material beyond this cross-over frequency is said to be solid-like. Entanglements of macromolecular solutions can result in such behavior. When $G^{\prime}$ is higher than $G^{\prime \prime}$ over most of the frequency range investigated, a weak gel behavior is observed due to the formation of a weak three-dimensional network of ordered chain segments.

Thus, viscoelastic structures of hydrocolloids may differ considerably. So, recent data about their behavior in mixtures with starch or model foods is discussed.

\section{Starch under heating}

Dynamic rheological tests allow continuous measurement of dynamic moduli during temperature and frequency sweep testing of a starch suspension. The rheological properties of starches differ, because their composition and granules' morphology is different. Thus, the identification of native starch sources is required in order to achieve the desired functionality and unique properties (Duxbury, 1989). Furthermore, concentration effects, temperature, heating rate and shear rate effects can be found and measured by rheological properties.

Concerning starch the following changes under heating can be measured using oscillatory experiments.

- Gelatinization

- Pasting

- Gelling and

- Retrogradation, which can be distinguished in short-term by network formation and long-term retrogradation that lasts several weeks

\subsection{Dynamic rheology and gelatinization}

During this first stage of heating, starch granules swell during the process of gelatinization. Soluble polymer molecules leach from the swollen granules and the rheological properties, such as storage modulus $\left(G^{\prime}\right)$ and loss modulus $\left(G^{\prime \prime}\right)$ of the starch increase to a maximum. A sharp increase in $G^{\prime}$ may occur between $60-80^{\circ} \mathrm{C}$ (Ahmed et al., 2008) caused by the formation of three-dimensional (3D) gel network developed by leached out amylose and reinforced by strong interactions among swollen starch particles (Fig. 1). Similar changes can occur when viscosity is measured.

The swelling of the granules is important for both viscosity increase and viscoeleasticity of the produced dispersions. Granules' morphology and rigidity, complexes with other components (e.g. lipid-amylose), amylose content, protein content are some factors that determine both peak values of the viscoelastic parameters and their breakdown thereafter.

Concerning their botanical source, among native starches (corn, rice, wheat and potato), potato starches exhibit the highest swelling power and final viscoelastic values. Their shape and size differs with respect to starches of other botanical sources. Starch granules of potato are smooth-surfaced and of different shapes form oval and irregular to cube-shaped. Starch 
granules of corn are angular-shaped, while those of rice are pentagonal and angular-shaped. Finally, wheat starch granules are spherical (B-granules) and lenticular-shaped (A-granules).

Moreover, potato starch granules are the largest $(<110 \mu \mathrm{m})$ in size followed by wheat $(<30 \mu \mathrm{m})$, corn $(<25 \mu \mathrm{m})$ and rice $(<20 \mu \mathrm{m})$ starches. The granule size of potato starch is variable and ranges form 1 to $20 \mu \mathrm{m}$ for small and from 20 to $110 \mu \mathrm{m}$ for large potato granules, whereas rice starch granules commonly range from 3 to $5 \mu \mathrm{m}$ in size (Hoover, 2001; Singh et al., 2003). Large and cubical or irregularly shaped granules in potato starch exhibit higher storage and loss modulus and lower tand than the small and oval granules (Singh \& Singh, 2001). Thus, potato starch shows higher $G^{\prime}, G^{\prime \prime}$ and lower tand than corn, rice and wheat starches during the heating cycle. Furthermore, starch dispersions may exhibit significantly higher $G^{\prime}$ values ( 100 times) as compared to flour dispersions at the first period of heating $\left(40-60^{\circ} \mathrm{C}\right)$ (Ahmed et al., 2008).

Amylose amount is also quite important for controlling the viscoelastic properties of starch dispersions. Amylose results in higher $G^{\prime}$ indicating a well-cross-linked nature. Specifically $\mathrm{G}^{\prime}$ can increase exponentially as a function of amylose content (Biliaderis \& Juliano, 1993). Concentration effects are also linear for wheat and maize starch in the range of $6-30 \%$ (Ring, $1985)$ and follow a power law in the case of rice starches (8-40\%).

Starch is a complicated viscoelastic structure. Under heating it can be described as a composite system, in which gelatinization may be regarded as an example of a phaseseparated composite gel, primarily governed by the volume fraction occupied by the swollen particles, whereas the continuous phase makes an additional contribution due to its own viscoelastic properties (Alloncle \& Doublier, 1991; Dickinson,1992).

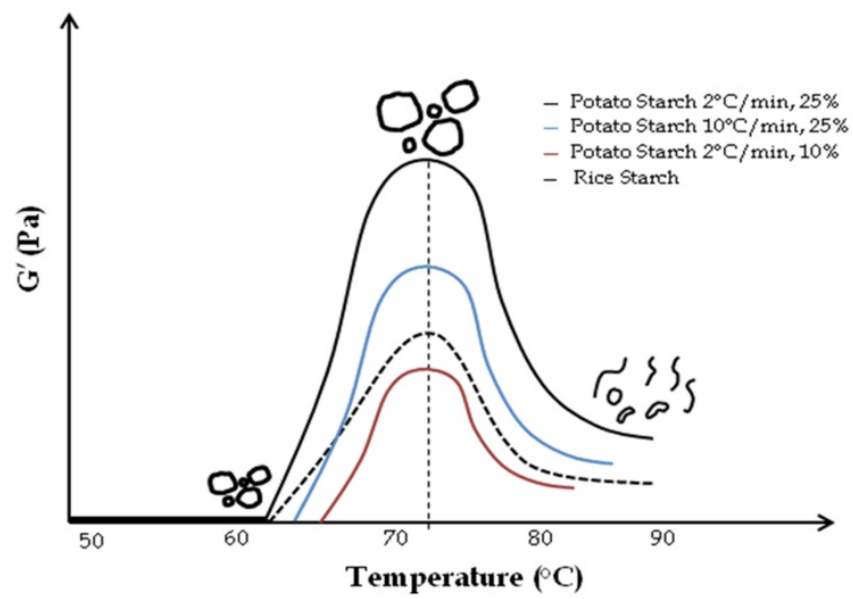

Figure 1. Viscoelastic changes of starch suspensions under heating and further cooling. Curves can shift to both axes accordingly to the factors that are mentioned. Changes in starch granules are also shown. Peak and plateau values can be seen at maximum starch swelling. Thereafter network breakdown and paste formation is shown. (Modification according to data from Ahmed et al., 2008, Food Hydrocolloids 22, pp 278-287 and Singh et al, 2007a, Starch/Stärke, 59, pp. 10-20) 


\subsection{Pasting and viscoelastic properties}

After gelatinization the process of pasting follows. Under continuing heating, granules are further swollen and finally disrupted (Fig.1). A hot paste is then created consisting of swollen granules, granule fragments, and soluble materials. The botanical source of the starch, water content, temperature and shearing during heating determine the consistency of this paste. The network created consists of dissolved starch polymers (amylose and amylopectin) and a discontinuous phase of swollen granules, empty (ghost) ones and fragments.

Rheologically, a peak value of both viscosity and $G^{\prime}, G^{\prime \prime}$ is reached resulting mainly from maximum swelling. Furthermore a plateau e.g. constant values may occur (from $80-85^{\circ} \mathrm{C}$ ) due to irreversible swelling and solubilisation of amylose (Ahmed et al., 2008) followed by a sudden drop of $G^{\prime}$ under extensive heating and shear and time. Granules disintegrate. At this point a hot paste is created (Fig. 1). The height of the peak at a given concentration reflects the ability of the granules to swell freely before their physical breakdown. A sudden drop after the maximum indicates the breakdown on cooking as well as a great ability to swell (Adebowale \& Lawal, 2003).

$G^{\prime}$ decrease indicates the gel structure disruption due to the "melting" of the crystalline regions or disentanglements of the amylopectin molecules in the swollen particles that softens the particles (Tsai et al., 1997). The network collapses due to the loss of interactions between the particles (Ahmend et al., 2008).

\subsection{Retrogradation and viscoelastic properties}

The distinction between a paste and a gel is not always evident. A paste usually refers to the hot freshly cooked system and gel is formed after cooling. Both are viscoelastic materials. As the hot pastes, especially of amylose -containing starches, begin to cool they become more elastic and develop solid properties. The transition from a viscous to an elastic gel can be determined by storage and loss moduli thus the setback can be found as a transition point from viscous to solid one (BeMiller, 2011). This setback is known as retrogradation (Atwell et al., 1988). At this critical gel point the system is wall-to-wall connected (percolation threshold) and is characterized by a critical behavior with $G^{\prime}(\omega)$ and $G^{\prime \prime}(\omega)$ obeying the same power law: $G^{\prime}(\omega) \sim G^{\prime \prime}(\omega) \sim \omega^{\text {n }}$ (Doublier \& Cuvelier, 2006).

The first phase of retrogradation begins as the paste cools and a formation of entanglements and/or junction zones is created between amylose molecules resulting in an elastic gel. This phase may last up to $48 \mathrm{~h}$. The second phase of retrogradation involves amylopectin changes, which is a much slower process that may proceed for several weeks depending on the storage temperature. Both $G^{\prime}$ and $G^{\prime \prime}$ increase upon cooling and during short-storage, $G^{\prime}$ and $G^{\prime \prime}$ increase indicating that the gels become firmer.

\section{Modified starches}

Briefly, chemical modification leads to a considerable change in the rheological and pasting properties of starches. Storage $\left(\mathrm{G}^{\prime}\right)$ and loss modulus $\left(\mathrm{G}^{\prime \prime}\right)$ of acetylated, hydroxypropylated 
and cross-linked starches from different sources increase to a maximum and then drop during heating following the same general rheological pattern as native starches (Singh et al., 2007b).

The temperature of maximum $\mathrm{G}^{\prime}$ drops significantly on acetylation or hydroxypropylation, while it increases after cross- linking (Kaur et al., 2004, 2006; Singh et al., 2004). Acetylated corn and potato starches showed greater values of $G^{\prime}$ and $G^{\prime \prime}$ under heating but lower compared to their native starch gels upon cooling of heated starch gels, confirming their lower tendency to retro gradate.

Strengthening bonding between starch chains by cross-linking will increase resistance of the granules towards swelling resulting in lower $G^{\prime}$ values in a high degree of cross-linking. Cross-link concentration and location could lead to different rheology. Botanical source also influences cross-linking and potato starches show a higher susceptibility towards crosslinking than other kinds of starches (Kaur et al., 2004).

\section{Interactions with other hydrocolloids}

According to an excellent review of BeMiller (2011) twenty-one different native starches in combination with thirty two different hydrocolloids have been investigated in different studies. Thus there is an increased interest in starch-hydrocolloid systems as well as a significant amount of scientific work in this area. New research works are mainly presented in this section, enhancing the knowledge about such systems.

\subsection{Hydrocolloids influence in starch pastes}

Starch pastes have typical biopolymer gel behavior. In typical biopolymer gel behavior greater $G^{\prime}$ values than $G^{\prime \prime}$ along the frequency sweep are observed, however in starch pastes both moduli are frequency dependent. Hydrocolloids modify the dynamic spectra of starch, although different trends can be observed.

First assumption: Hydrocolloids lead to weaker structures with less gel-like character.

With their addition, starch network shifts from an elastic-like to a more viscous-like one (Rosell et al., 2011). Starch-hydrocolloid systems can be considered as biphasic systems. When starch granules are swollen, the hydrocolloid is located entirely in the continuous phase. The concentration of hydrocolloid will then increase as the volume of the phase accessible to the hydrocolloid is reduced. This fact changes the viscoelasticity of the starch. Thus, cellulose derivatives and carrageenans can lead to less solid-like pastes than the control paste (Techawipharat et al., 2008). This assumption depends on starch type as well. In waxy starches, due to an absence of amylose, short-term retrogradation does not occur and therefore, the addition of hydrocolloids could not alter the viscoelastic characteristics of these starch pastes.

Second assumption: Hydrocolloids addition leads to associations with starches resulting in increased $G^{\prime}, G^{\prime \prime}$ values. 
Several hydrocolloids can promote associations with starches and as a result, when they are added in starch pastes, an increase in $G^{\prime}, G^{\prime \prime}$ is often observed, see examples: Mandala et al.(2004a); Achayuthakan \& Suphantharika, (2008); Wang et al., (2008). In such systems the question is which ingredient predominates in the overall rheology, starch or hydrocolloid. In wheat starch-hydrocolloid systems, it is the hydrocolloid that predominates in the whole system, according to the shift factors found.
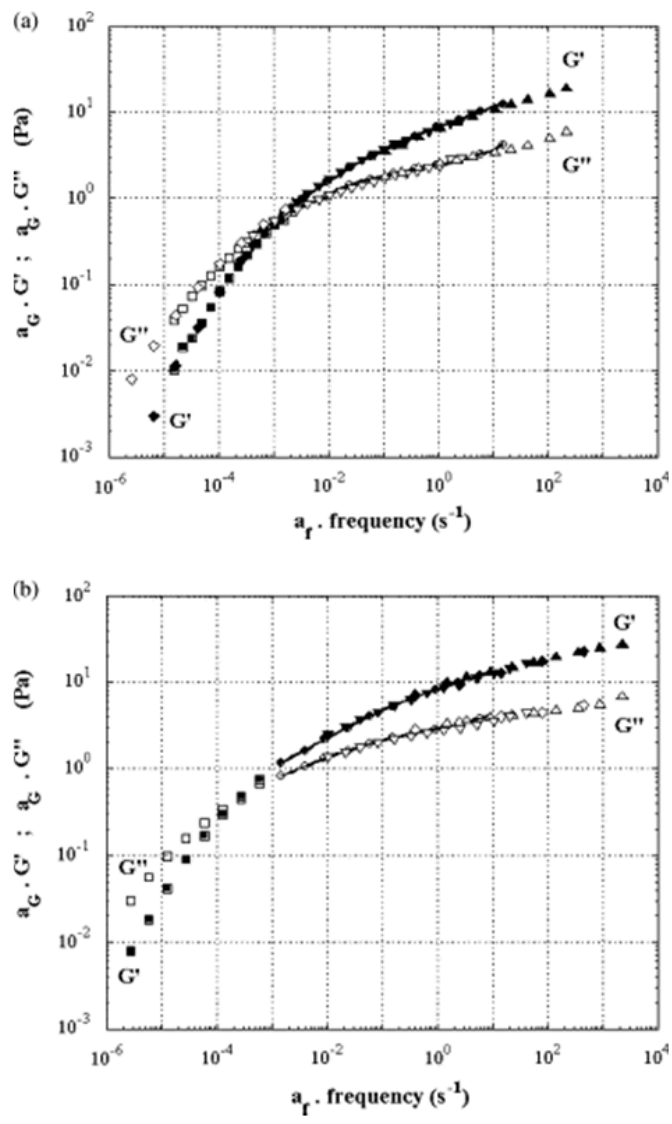

(a) In water of xanthan $0.1 \mathrm{wt} \%(\square, \mathbf{\square})$ and $0.5 \mathrm{wt} \%(\circ, \bullet)$, starch $2 \mathrm{wt} \% /$ xanthan $0.5 \mathrm{wt} \%(\Delta, \mathbf{\Delta})$, amylose 0.3 wt $\% /$ xanthan $0.1 \mathrm{wt} \%(\bullet, \bullet)$, amylose $0.3 \mathrm{wt} \% /$ xanthan $0.5 \mathrm{wt} \%(\nabla, \mathbf{\nabla})$.

(b) In $0.1 \mathrm{M} \mathrm{NaCl}$ of xanthan $0.1 \mathrm{wt} \%(\square, \mathbf{\square}), 0.5 \mathrm{wt} \%(\circ, \bullet)$ and $0.8 \%(\bullet, \bullet)$, starch $2 \mathrm{wt} \% /$ xanthan $0.5 \mathrm{wt} \%(\Delta, \mathbf{\Delta})$, amylose $0.3 \mathrm{wt} \% /$ xanthan $0.5 \mathrm{wt} \%,(\boldsymbol{\nabla}, \boldsymbol{\nabla})$. (From Mandala et al, 2004a. Carbohydrate Polymers 58, pp 285-292, with permission).

Figure 2. Superimposed shifted spectra.

Furthermore, except $G^{\prime}, G^{\prime \prime}$ values, tan $\delta$ may be important in interpretations of the behavior of starch-hydrocolloids interactions. Thus, although some hydrocolloids promote an increase in $G^{\prime}, G^{\prime \prime}$ by their addition, in fact they lead to a less solid-like system (higher tano values) as described above. On the contrary, in some cases, hydrocolloid addition may lead 
to a more solid-like system as noticed in systems of maize starch with flaxseed gum (Wang et al., 2008).

In this research work, the variation of the $G^{\prime}$ with frequency for the maize starch alone and the flaxseed gum-maize starch mixtures with different flaxseed gum concentrations was not significant. This suggests that both the maize starch and its mixture with flaxseed gum have a typical biopolymer gel network, but flaxseed gum helps the formation of stronger gels. Concerning temperature effects, at a temperature range of $25-75^{\circ} \mathrm{C}$, flaxseed gum addition shows more significant temperature dependence compared to that of maize starch alone. An increase in temperature results in a decrease in $G^{\prime}$ of the mixture, indicating that the addition of flaxseed gum affects the thermal stability of the mixture (Wang et al., 2008).

\subsection{Influence of hydrocolloids during storage}

Gelation and short- or long-term retrogradation of starch can be influenced by hydrocolloids. The addition of a hydrocolloid can accelerate gelation and reduce retrogradation (Kim \& Yoo, 2006, Lee et al., 2002; Mandala \& Palogou, 2003; Fumami et al., 2005,2008 ) but this depends on many parameters, some of which are discussed extensively in the following text.

Concerning gelation, starch-hydrocolloid mixtures may display weak gel-like behavior (Funami et al., 2008; Kim \& Yoo, 2006; Lee at al., 2002). According to time-dependent curves of the mixtures of rice starch-xanthan gum, $G^{\prime}$ values increase rapidly during the first few hours at low temperature aging $\left(5^{\circ} \mathrm{C}\right)$ and remained steady afterwards. Gelation could be considerably shortened by the presence of xanthan gum. Increasing xanthan gum concentration increased $G^{\prime}$ values during aging, indicating that the elastic character of xanthan gum influences the reinforcement of the overall gel properties during aging. A rapid increase and subsequent plateau of $G^{\prime}$ can be shown by xanthan gum addition (Fig. 3). This is due to the rapid aggregation of amylose chains at the early stage and the slow aggregation of amylopectin chains at the late stage respectively (Kim \& Yoo, 2006).

First-order kinetics for structure development of starch-xanthan mixtures during aging (recrystallization) and further retarding during longer storage can be developed. The rate of $\mathrm{G}^{\prime}$ increase (structure development) due to the retrogradation of rice starch during cold storage is apparently affected by the presence of xanthan gum and greatly dependent on the xanthan gum concentration. The same was observed for wheat starch-xanthan mixtures aging (Mandala \& Palogou, 2003).

In the long-term retrogradation of different hydrocolloid-starch mixtures (Funami et al., 2008; Kim \& Yoo, 2006; Lee at al., 2002) molecular associations between the gum and the amylopectin fraction inhibit the formation of crystalline structures during storage. As another factor to inhibit long-term retrogradation, gums can stabilize water molecules, therefore they can act as water binder effectively depriving amylose or amylopectin of usable water for crystallization as described in the case of corn starch fenugreek gum mixtures (Funami et al., 2008). 


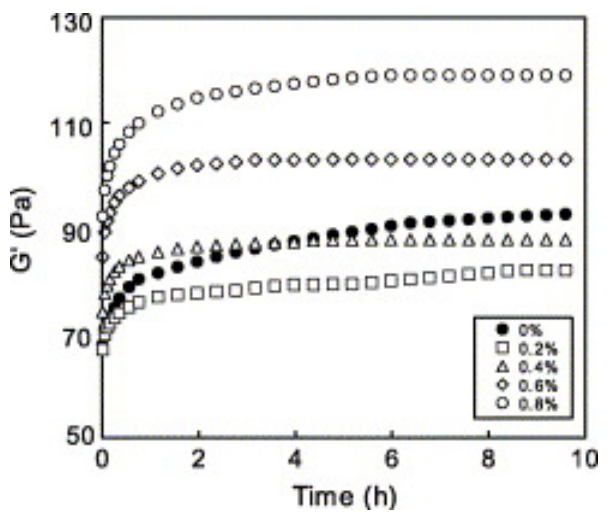

Figure 3. Changes in storage modulus $\left(\mathrm{G}^{\prime}\right)$ during aging at $4^{\circ} \mathrm{C}$ for $10 \mathrm{~h}$. Close symbol rice starch. Open symbols rice starch-xanthan gum mixtures (XG:0.2-0.8\%). (From Kim \& Yoo, 2006, Journal of Food Engineering 75, pp. 120-128, with permission).

Gelation and retrogradation can be also influenced by the molecular size of the hydrocolloid in a starch-gum mixture. Thus, the molecular mass and size of guar gum influences gelatinization and retrogradation behaviour of corn starch according to Funami et al. (2005a, 2005b). Viscosity and viscoelastic properties can be measured. Molecular interactions between guar gum and amylose are responsible for an earlier onset of viscosity increase for the composite system of starch-guar gum, whereas molecular interactions between guar gum and amylopectin are responsible for the increase in peak viscosity of the composite system. Moreover, the addition of guar gum accelerates the gelation of starch, in particular when the amylose fraction increases. Concerning the control of retrogradation by adding guar gum, storage modulus $\left(G^{\prime}\right)$ for starch systems increases rapidly at very early stage of storage at $4^{\circ} \mathrm{C}$.

Short-term retardation of retrogradation is also suggested, because the gelled fraction in the system is reduced with the addition of guar gum (loss targent increase). This happens due to the decrease in the amount of amylose leached out of the starch granules during gelatinization. There is a critical $\mathrm{Mw}$ up to which the amount of leached amylose can be influenced, which is $15.0 \times 10^{5} \mathrm{~g} / \mathrm{mol}$. The effect of guar gum on the inhibition of short-term retrogradation becomes less $\mathrm{Mw}$-dependent at above this $\mathrm{Mw}$ value. On the other hand, the higher the Mw of guar gum, the easier the guar interacts with amylopectin.

$\mathrm{G}^{\prime}$ becomes less-frequency dependent with decreasing $\mathrm{Mw}$ of guar gum. These results suggest that the interactions between guar gum and amylose should hardly contribute to forming a gelled or ordered structure (Funami, 2005). Furthermore, the ability of guar gum to inhibit long-term retrogradation is enhanced markedly when the $\mathrm{Mw}$ of the guar is over $30.0 \times 10^{5} \mathrm{~g} / \mathrm{ml}$. Thus, above this molecular weight guar gum can act easily on either amylose or amylopectin to retard starch crystallization.

Concluding:

- Hydrocolloid addition may decrease or increase the gel-like character of starch pastes depending on hydrocolloid and starch type as well as on gum concentration. The most 
common observation was the increase in both viscous and elastic character with more pronounced effects on the viscous one.

- Regarding gelation, it is induced by adding hydrocolloids. Short-term retrogradation is also related to this gelation acceleration, as well as to amylose amount.

- Gelation acceleration does not mean retrogradation acceleration as well, since a more viscous character is maintained by hydrocolloid addition in many cases.

- Concerning long-term retrogradation, it is clearly reduced by hydrocolloid addition. Factors that may contribute are the associations of hydrocolloid-amylopectin, the stabilization of water molecules and last but not least the molecular weight of the gum.

\section{Applications to foodstuffs}

In a food system, many other ingredients influence the process of gelatinization, pasting, breakdown and retrogradation/setback of starch pastes (BeMiller, 2011). Recent data about starch pastes and gels as governed by their ingredients and the interactions with other hydrocolloids is presented. Furthermore, the role of these interactions in controlling rheology in model food is discussed. Examples given include stability issues of ready to eat white sauces, soups and caramel sauces.

\subsection{White sauces}

Béchamel sauce or "white sauce" is used in a lot of preparations or as a basis for other more complex sauces (Heyman et al., 2010). Sauces often exhibit stability problems during prolonged storage either caused by emulsion instability or by changing polymer interactions (Mandala et al., 2004b; Mc Clemments, 2006).

Non-starch hydrocolloids added in starch paste can alter the continuous phase of the system which contains them. In a complex system like that of a sauce, apart from rheology, they can also alter water holding capacity of the sauces. The effects of partially replacing modified starch by hydrocolloids (guar gum, xanthan gum and carboxymethylcellulose) on the rheological behavior and the physicochemical stability of the sauces is discussed.

Oscillatory spectra of all sauces are very similar to those of starch gels (example model sauce and guar gum at different concentrations, (Fig. 4)). All samples exhibit a dominant elastic behavior since $G^{\prime}$ is larger than $G^{\prime \prime}$ over the studied frequency range.

When different hydrocolloids such as xanthan, guar or CMC gum are added, xanthan gum causes the greatest increase in $\mathrm{G}^{\prime}$ compared to the model system. Guar gum and CMC also shift the G' curve to higher values (Fig.4), but in a less pronounced way than xanthan gum does. White sauces present similarities between their values and those of starch gels alone supporting the hypothesis of a strong influence of both native and modified starches present in the system (Caisawang \& Suphantharika 2006). Same conclusions are reached by Mandala et al. (2004b). During preservation at $7^{\circ} \mathrm{C}$ for 30 days the overall profile of the frequency curves does not change. Slight reductions in both $G^{\prime}$ and $G^{\prime \prime}$ are noticed with the most significant changes to occur the first 2 weeks. 


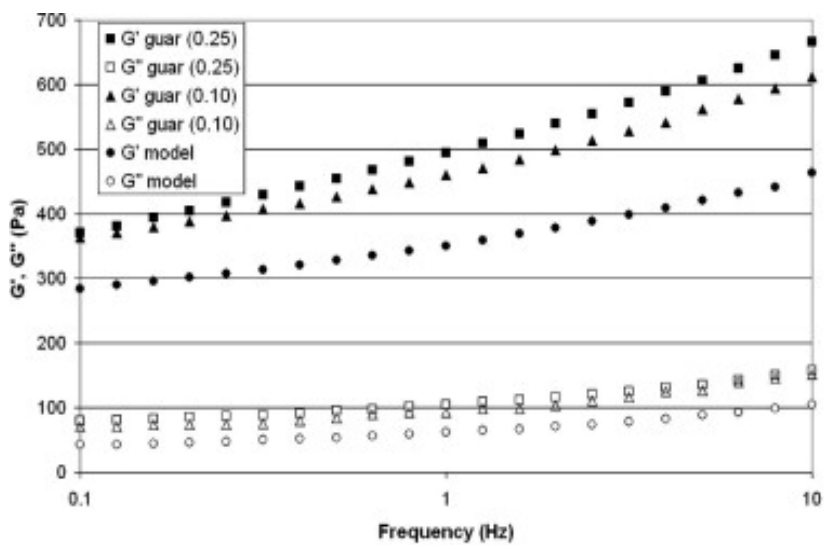

Figure 4. Effect of guar addition on oscillatory measurement of béchamel sauces. (From Heyman et al. (2010). Journal of Food Engineering 99, 115-120, with permission).

\subsubsection{White sauce and freeze-thaw stability}

Starches combined with different hydrocolloids are used in white sauces and the freeze/thaw stability of the produced samples is investigated. In a typical white sauce, after a freeze/thaw cycle, an increase in the viscoelastic functions is observed as a consequence of extensive starch retrogradation. By adding hydrocolloids this increase is reduced, leading to a less structured system. This can be justified by hydrocolloid interaction with solubilised amylose that reduces amylose - amylose interactions, preventing also structure ordering and hence reducing the extent of retrogradation (Arocas et al., 2009).

\subsubsection{Ambient conditions' thawing}

Specifically, the viscoelastic properties of fresh and thawed white sauces containing different corn starches (native waxy corn starch (NWS), native corn starch (NS), hydroxypropyl distarch phosphate waxy corn starch (HPS) and pregelatinized acetylated distarch adipate waxy corn starch (AAS)) are compared. Samples are frozen at $-18^{\circ} \mathrm{C}$ and thawed at room temperature until $20^{\circ} \mathrm{C}$.

A different behavior is found among the modified and the native starch sauces (Fig. 5).

The fresh modified starch sauces show higher $G^{\prime}$ and $G^{\prime \prime}$ values than the fresh native starch sauces, HPS being the one with the highest capacity and NWS the one with the lowest capacity. A high thickening capacity is ascribed to the fact that modified starches present high starch granule stability in comparison to the native starches and their granules do not break down in the thermal and shear conditions.

Moreover, a temperature increase from 20 to $80^{\circ} \mathrm{C}$ does not affect the values of $\mathrm{G}^{\prime}$ and $\mathrm{G}^{\prime \prime}$ either in the fresh or freeze/thaw samples. On the contrary, in native starch sauces a slight decrease in the values of the viscoelastic moduli is observed after $50^{\circ} \mathrm{C}$, particularly pronounced after the freeze/thaw cycle. Furthermore, the values of the $G^{\prime}, G^{\prime \prime}$ of samples 
prepared with native starches after freeze/thaw cycle are much greater than those of the fresh samples due to retrogradation phenomena occurring during the freezing process.
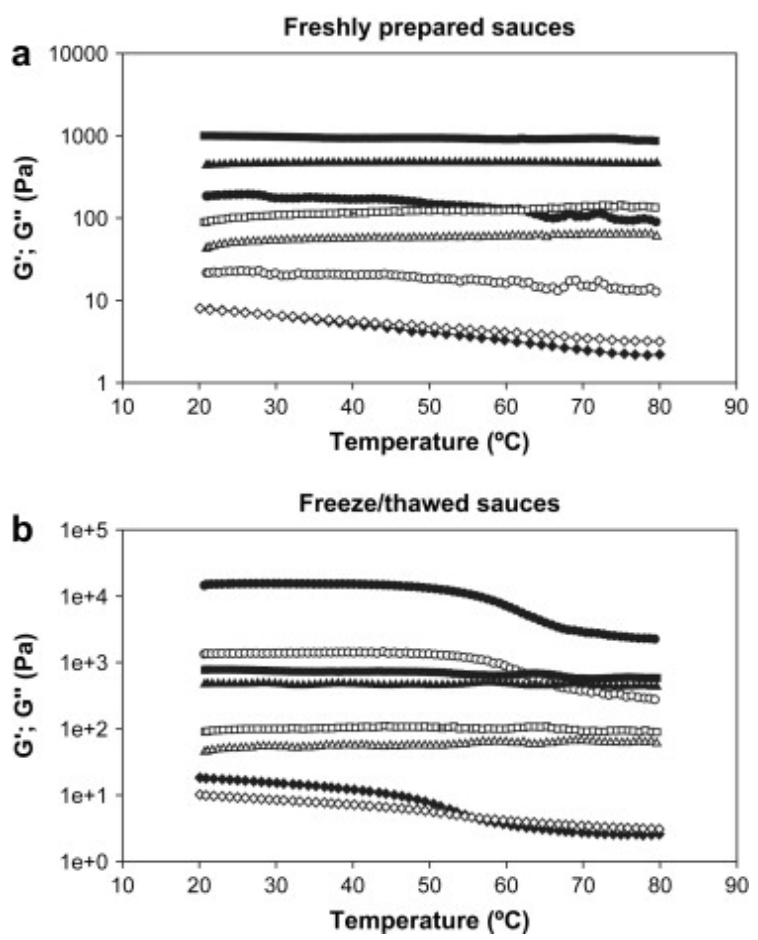

Figure 5. $G^{\prime}$ and $G^{\prime \prime}$ as a function of increasing temperature for : a) freshly prepared sauces and b) freeze/thawed sauces. NS sauce $\left(G^{\prime}: \bullet, G^{\prime \prime}: \circ\right)$, NWS sauce $\left(G^{\prime}: \bullet, G^{\prime \prime}: \diamond\right)$, AAS sauce $\left(G^{\prime}:\left(\boldsymbol{\Lambda}, G^{\prime \prime}: \Delta\right)\right.$ and HPS sauce $\left(G^{\prime}: \mathbf{-}, G^{\prime \prime}: \square\right)$. Frequency: 1 Hz. $\gamma: 0.001$. Heating rate: $1.5^{\circ} \mathrm{C} / \mathrm{min}$. (From Arocas et al., 2009, Food Hydrocolloids, 23, pp 901-907, with permission).

Concerning mechanical spectra, all samples behave as soft gels with values of $G^{\prime}$ higher than values of $G^{\prime \prime}$. A weak dependence on frequency is observed, as well as in starchhydrocolloid mixtures. After freeze/thaw, as it was expected, structural changes occur mainly in sauces containing native starches. As a consequence, great values of $\mathrm{G}^{\prime}$ and $\mathrm{G}^{\prime}$ are observed and these samples presented a spongy structure depending also on freezing rate (the lower the freezing rate, the more pronounced the spongy structure). Thus, chemical modification is effective in providing freezing and thermal structure stability.

\subsubsection{Microwave and water bath thawing}

Differences between conductive heating and MW heating of starch dispersions are found not in the mechanism of gelatinization but on the starch crystallinity, which disappears at a higher rate when samples were heated by microwaves. Furthermore, the attainment of a certain viscosity required longer time in the conduction-heated samples. Microwaving can cause incomplete gelatinization of the starch in comparison to 
convection heating and potato starch can be influenced much more than corn starches (An et al., 2008).

The effects of microwave thawing and water bath thawing on white sauces prepared with two different native starches (potato and corn) and a modified waxy maize starch are compared. Starch retrogradation is strongly affected by freezing and thawing. Thus, possible reduction of starch retrogradation upon different thawing methods could be beneficial for the quality characteristics of the final product.

Furthermore, microwave treated samples are quite similar to the freshly prepared sauces compared to the water bath-thawed ones. In this regard, microwaving could be considered more suitable than the water bath for diminishing the loss of quality associated with the freezing step (Table 1).

The differences in the viscoelastic properties of the microwave-and water bath-thawed native starch sauce can be explained because of the shorter heating time required in the microwave, which reduces the time available for the retrogradation occurring from -5 to $20^{\circ} \mathrm{C}$. Furthermore, the big local temperature and the differences occurring during microwave heating can lead to an improved localized melting of amylopectin and eventual melting of amylose (at temperatures near $100^{\circ} \mathrm{C}$ ).

\begin{tabular}{|c|c|c|c|c|}
\hline Starch type & Treatment & $\mathrm{G}^{\prime} \mathbf{( P a )}$ & $\left.\mathrm{G}^{*} \mathbf{( P a}\right)$ & $\tan \boldsymbol{8}$ \\
\hline \multirow{4}{*}{ Corn } & $\mathrm{WB}$ & $326.0 \mathrm{~A}$ & $328.0 \mathrm{~A}$ & $0.114 \mathrm{C}$ \\
\cline { 2 - 5 } & $\mathrm{MW}$ & $265.2 \mathrm{~B}$ & $267.0 \mathrm{~B}$ & $0.118 \mathrm{C}$ \\
\cline { 2 - 5 } & Fresh & $80.4 \mathrm{E}$ & $81.9 \mathrm{E}$ & $0.189 \mathrm{~B}$ \\
\hline \multirow{4}{*}{ Potato } & $\mathrm{WB}$ & $177.6 \mathrm{C}$ & $179.8 \mathrm{C}$ & $0.158 \mathrm{BC}$ \\
\cline { 2 - 5 } & $\mathrm{MW}$ & $114.7 \mathrm{D}$ & $116.7 \mathrm{D}$ & $0.183 \mathrm{~B}$ \\
\cline { 2 - 5 } & Fresh & $97.6 \mathrm{DE}$ & $101.0 \mathrm{DE}$ & $0.265 \mathrm{~A}$ \\
\hline \multirow{4}{*}{ Modified waxy corn } & $\mathrm{WB}$ & $270.7 \mathrm{~B}$ & $272.5 \mathrm{~B}$ & $0.118 \mathrm{C}$ \\
\cline { 2 - 5 } & $\mathrm{MW}$ & $264.7 \mathrm{~B}$ & $267.7 \mathrm{~B}$ & $0.149 \mathrm{BC}$ \\
\cline { 2 - 5 } & Fresh & $206.6 \mathrm{C}$ & $210.5 \mathrm{C}$ & $0.194 \mathrm{~B}$ \\
\hline
\end{tabular}

Table 1. Influence of starch type and treatment in the rheological parameters $\mathrm{G}^{\prime}, \mathrm{G}^{*}$ and $\tan \delta$. Frequency: $1 \mathrm{~Hz}$. ABCDE Means with the same letter are not significantly different $(\mathrm{p}<0.05)$ according to the Tukey's multiple range test. WB water bath thawed samples, MW microwave thawed samples. (From Arocas et al, 2011, Food Hydrocolloids 25, pp 1554- 1562, with permission).

This can lead to new starch/water interactions and consequent water adsorption. Generally, heating during thawing improves the quality of frozen sauces as amylose bonds formed during retrogradation are broken accompanied with re-absorption of the previously released water. Furthermore, the modified starch resists the heating applied during the 
sauce preparation as well as during the thawing process. Although the modified starch granules swell, little release of the starch components occurs. Thus, the differences between thawing techniques are related to their effect on structure changes related to starch retrogradation (Arocas et al., 2011).

\subsection{Chilli sauce}

Chilli sauces investigated presented a dominant elastic behavior compared to the viscous behavior typically observed in suspensions with network-like structure (Gamonpilas et al., 2011). Weak gel-like characteristics are found in chilli sauces containing starch and hydrocolloids, as well as in white sauces mentioned above. The presence of starch/xanthan mixture in the commercial chilli sauces promotes their elastic properties. Furthermore, the sauce with low solid content and without xanthan gum has weak network structure and inferior flow properties. The addition of xanthan gum and/or modified starch can provide a network-like characteristic of the sauce.

\subsection{Soup formulation}

In a soup formulation corn starch was gradually replaced with fenugreek gum (FG) till $0.9 \%$ wt. Pure systems of corn starch and fenugreek gum dispersions are also tested. Pure corn starch-FG mixtures and soups are subjected to rheological measurements after preparation. (Matia-Merino \& Ravindran, 2009).

Final viscosities increase with increasing FG replacement levels. Soup containing FG (without starch) exhibit a dose-related pasting pattern and different pasting profiles than starch-FG soup formulations. Combined addition of starch-FG results in a synergistic effect of starch and FG. Thus, the viscosity increase is much greater than when the two ingredients are added individually. The viscoelasticity of the starch-FG systems and soups containing them is shown at the frequency of $1 \mathrm{~Hz}$ (Fig 7.a, Fig 7b). The viscous or loss modulus shows slightly more dependency with the elastic component being frequently independent and always over the viscous modulus through the entire frequency range. The main differences between starch-FG systems and soups containing them is: a) the higher viscoelasticity developed in all the soups with both $G^{\prime}$ and $G^{\prime \prime}$ being greater in value than in the pure starch/fenugreek mixtures, measured under similar conditions and b) both elastic and viscous moduli gradually increase with the substitution of starch by FG for all formulations.

\subsection{Caramel sauces}

Caramel sauces can have tailored sensory, rheological and textural characteristics by using potato starch and xanthan gum combinations (Krystyjan et al., 2012). Potato starch and xanthan gum are selected among other starch-hydrocolloid combinations taking into account transparency and clear appearance for further use in caramel sauces as thickeners. Commercial sauce without any stabilisers received the lowest score in sensory evaluation. Samples were stored and among other rheological characteristics, storage and loss moduli 

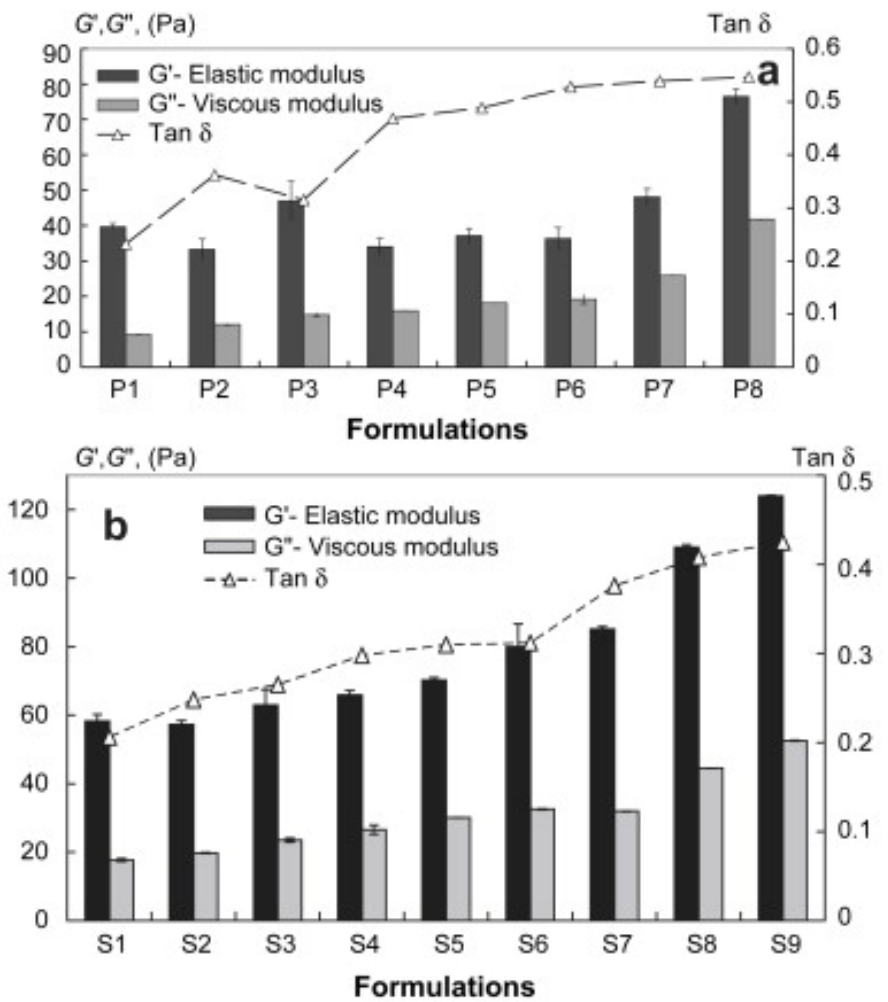

(a) 5\% standard corn starch (P1), gradually replaced with fenugreek at $0.1 \%$ (P2), $0.15 \%$ (P3), 0.2\% (P4), 0.25\% (P5), $0.3 \%$ (P6), 0.5\% (P7), 0.7\% (P8), 0.9\% (P9).

(b) soup formulations with $5 \%$ standard corn starch (S1), gradually replaced with fenugreek at $0.1 \%$ (S2), $0.15 \%$ (S3), $0.2 \%$ (S4), $0.25 \%$ (S5) and $0.3 \%$ (S6), $0.5 \%$ (S7), $0.7 \%$ (S8), $0.9 \%$ (S9). All measurements were carried out at $25^{\circ} \mathrm{C}$ (From Matia-Merino \& Ravindran, 2009, Food Hydrocolloids, 23(3), pp 1047-1053, with permission).

Figure 6. Storage modulus $\left(G^{\prime}\right)$, loss modulus $\left(G^{\prime \prime}\right)$, and $\tan \delta$ measured at $1 \mathrm{~Hz}$ and $1 \%$ strain

were measured. On the contrary to the assumptions in previous examples, the storage moduli of all sauces are much lower than their loss moduli. Sauces have very weak elastic and very strong plastic properties. This feature is considered disadvantageous, because the sauce can very easily flow down from the surface of the glazed products. An increase in xanthan gum amount results in moduli increase, both before and after storage. Controlled rheology can be achieved improving caramel sauces performance.

Concluding:

- Sauces and soups containing starch and hydrocolloids behave like weak gel-like products with good network structure.

- Caramel sauces containing starch and hydrocolloids are fluid-like with viscous character much more pronounced than the elastic one. 
- Viscocelastic behavior of soups is similar to that of simple hydrocolloid-starch systems. However, viscous-like behavior is more pronounced in final soups than in starchhydrocolloids' systems.

- Thawing under heating of frozen ready to eat starch white sauces results in improved rheological characteristics of the final samples. In particular, thawing under microwaves leads to better products than those being thawed in a water bath.

\section{Conclusions}

In this chapter oscillatory rheological data are presented to highlight the structural changes of starch suspensions during heating and further storage. Furthermore, the viscoelastic behavior of different starch types with selected hydrocolloids is presented in order to understand and control rheology in food processing. Moreover, the rheological behavior of starch-hydrocolloid mixtures is described in complex systems, they contain them that aim at successful food product development.

Starches undergo significant changes under heating that are related to structural changes and can be determined using small amplitude oscillatory shear (SAOS) tests. In many research works these tests are also combined with rapid visco analyser (RVA measurements), viscosity values, texture analyses, microscopic observations, DSC thermographs.

Hydrocolloids alter the viscoelastic character of starch pastes and different mechanisms are proposed, some of them based on their interactions with starch polymers. Hydrocolloids are found in the continuous phase of the starch pastes changing the effective concentration of starch in the final mixtures. They can also immobilize the water available, or they can interact with starch polymers. Pastes containing hydrocolloids may be less structured and less solid-like than the control pastes. Specific interest presents their role in gelation and retrogradation, since they are considered anti-staling agents and can prohibit retrogradation effects, specifically those of amylopectin.

Controlled rheological properies can be achieved by changing hydrocolloid-starch mixtures in selected products such as sauces, soups and caramel sauces, e.g. the final quality of native starches white sauces can be maintained when appropriate thawing process under fast heating (microwaves) is used.

New kinds of hydrocolloids are going to be used and starch role especially that of native starches is re-defined. The interest about such systems is going to increase and research on this field will be continued.

\section{Author details}

Ioanna G. Mandala

Agricultural University Athens, Dept. Food Science and Technology,

Greece 


\section{References}

Adebowale, K. O., \& Lawal, O. S. (2003). Functional properties and retrogradation behaviour of native and chemically modified starch of mucuna bean (Mucuna pruriens). Journal of the Science of Food and Agriculture, 83, pp. 1541-1546.

Achayuthakan P. \& Suphantharika M. (2008). Pasting and rheological properties of waxy corn starch as affected by guar and xanthan gum. Carbohydrate Polymers, 71, pp. 9-17

Arocas A., Sanz T. \& Fiszman S.M. (2009). Influence of corn starch type in the rheological properties of a white sauce after heating and freezing. Food Hydrocolloids, 23, pp. 901907

Acoras A., Sanz T., Hernando M.-I. \& Fiszman S.M. (2011). Influence of corn starch type in the rheological properties of a white sauce after heating and freezing. Food Hydrocolloids, 23, pp. 901-907

Ahmed J., Ramaswamy H. S., Ayad A. \& Alli I. (2008). Thermal and dynamic rheology of insoluble starch from basmati rice. Food Hydrocolloids 22, pp. 278-287

An, H.J., Yang H.S., Liu Z.D. \& Zhang Z.Z. (2008). Effects of heating modes and sources on nanostructure of gelatinized starch molecules using atomic force microscopy. LWTFood Science \&Technology 41, pp. 1466-1471

Alloncle M. \& Doublier J.-L. (1991). Viscoeleastic properties of maize starch/hydrocolloid pastes and gels. Food Hydrololloids, 5(5), pp. 455-467

Atwell, W. A., Hood, L. F., Lineback, d. R., Varriano-marston, E., \& Zobel, H. F. (1988). The terminology and methodology associated with basic starch phenomena. Cereal Foods World, 33, pp. 306-311.

BeMiller J.N. (2011). Pasting, paste, and gel properties of starch-hydrocolloid combinations. Carbohydrate Polymers 86, pp. 386-423

Bilbao-Sainz C., Burtler M., Weaver T. \& Bent J. (2007). Wheat and starch gelatinization undermicrowave irradiation and conduction heating. Carbohydrate Polymers 69, pp 224-232

Biliaderis C., Juliano B. (1993). Thermal and mechanical properties of concentrated rice starch gels of varying composition. Food Chemistry 48, pp. 243-250

Bowler P., Williams M.R. \&AngoldR.E. (1980). A hypothesis for the morphological changes which occur on heating lenticular wheat starches in water. Starch/Stärke, 34, pp. 149

Chaisawang M. \& Suphantharika M. (2006). Pasting and rheological properties if native and anionic tapioca starches as modified by guar gum and xanthan gum. Food Hydrocolloids 20 (5), pp. 641-649

Dickinson E. 1992. Rheology In: An introduction to food colloids, Oxford University Press, pp 59-62.

Doublier J.-L. \& Cuvelier G. (2006). Gums and Hydrocolloids: functional aspects in Carbohydrates in food, ed. A.-C. Eliasson, CRC: Taylor \&Francis, London 
Duxbury D.D. (1989). Modified starch functionalities-no chemicals or enzymes. Food Processing, 50 , pp. 35-37

Ferry J.D. (1980). Viscoelastic properties of polymers (3rd ed.). J. Wiley and Sons, New York

Funami T., Kataoka Y., Omoto T., Goto Y., Asai I. \& Nishinari K. (2005). Food hydrocolloids control the gelatinization and retrogradation behavior of starch. 2a: Function of guar gum with different molecular weights on the gelatinization behavior of corn starch. Food Hydrocolloids 19, pp. 15-24

Funami, T., Kataoka, Y., Noda, S., Hiroe, M., Ishihara, S., Asai, I., Takahashi R.\& Nishimari K. (2008). Functions of fenugreek gum with various molecular weights on the gelatinization and retrogradation behaviors of corn starch-1: Characterizations of fenugreek gum and investigations of corn starch/fenugreek gum composite system at a relatively high starch concentration; 15w/v\%. Food Hydrocolloids, 22, pp. $763-$ 776.

Funami T. (2011). Next target for food hydrocolloid studies: Texture design of foods using hydrocolloid technology. Food Hydrocolloids, 25(8), pp. 1904-191

Gamonpillas C., Pongjaruvat W., Fuonfuchat A., Methacanon P., Seetapan N. \& Thamjedsada N. (2011). Physicochemical and rheological characteristics of commercial chilli sauces as thickened by modified starch or modified starch/xanthan mixture. Journal of Food Engineering 105, pp. 233-240

Heyman B., Depypere F., Delbaere C. \& Dewettinck K. (2010). Effects of non-starch hydrocolloids on the physicochemical properties and stability of a commercial béchamel sauce. Journal of Food Engineering 99 ,pp. 115-120

Hoover R. (2001). Composition, molecular structure, and physicochemical properties of tuber and root starches: a review. Carbohydrate Polymers 45, pp. 253-267

Kaur, L., Singh, N., \& Singh, J. (2004). Factors influencing the properties of hydroxypropylated potato starches. Carbohydrate Polymers, 55, pp. 211-223

Kaur, L., Singh, J., \& Singh, N. (2006). Effect of cross-linking on some properties of potato starches. Journal of the Science of Food and Agriculture 86, pp. 1945-1954

Kim C. \& Yoo B. (2006). Rheological properties of rice starch-xanthan gum mixtures. Journal of Food Engineering 75, pp. 120-128

Krystyjan M., Sikora M., Asamczyk G. \& Tomasik P. (2012). Caramel sauces thickened with combinations of potato starch andxanthan gum. Journal ofFoodEngineering 112, pp 2228

Lee M.H., Baek M.H., Cha D.S., Park H.J. \& Lim, S.T. (2002). Freeze-thaw stabilization of sweet potato starch gel be polysaccharide gums. Food Hydrocolloids, 16, pp. 345352

Mandala I. \& Palogou E. (2003). Effect of Preparation Conditions and Starch/Xanthan Concentration on Gelation Process of Potato Starch Systems. International Journal of Food Properties 6, pp. 311-328 
Mandala I. G., Michon C. \& Launay B. (2004a). Phase and rheological behaviors of xanthan/amylose and xanthan/starch mixed systems. Carbohydrate Polymers 58, pp. 285-292

Mandala I.G., Savvas T.P., Kostaropoulos A.E. (2004b). Xanthan and locust bean gum influence on the rheology and structure of a white model-sauce. Journal of Food Engineering 64(3), pp. 335-342

Mc Clemments D.J. (2006). Non-covalent interactions between proteins and polysaccharides. Biotechnology Advances 24(6), pp. 621-625

Matia-Merino L. \& Ravindran G. (2009). Starch-fenugreek (Trigonella foenum-graecum L.) polysaccharide interactions in pure and soup systems, Food Hydrocolloids, 23(3), pp. 1047-1053

Rosell C.M., YokoyamaW. \& Shoemaker C. (2011). Rheology of different hydrocolloids-rice starch blends. Effect of successive heating-cooling cycles. Carbohydrate Polymers 84, pp. 373-382

Ross-Murphy S.B. (1984). Rheological methods, Biophysical Methods in Food Research. vol.5 (H.W.-S. Chan, ed.) SCI Critical Reports on Applied Chemistry, Blackwell, Oxford, pp. 137-139

Singh, J., \& Singh, N. (2001). Studies on the morphological, thermal andrheological properties of starch from some Indian potato cultivars. Food Chemistry, 75, pp. 6777

Singh N., Singh J., Kaur L., Singh S. N. \& Singh G. B. (2003). Morphological, thermal and rheological properties of starches from different botanical sources. Food Chemistry 81 (2), pp. 219-231

Singh, N., Chawla, D., \& Singh, J. (2004). Influence of acetic anhydride on physicochemical, morphological and thermal properties of corn and potato starch. Food Chemistry, 86, pp. 601-608.

Singh N., Nakaura Y., Inouchi N. \& Nishinari K. (2007a). Fine structure, thermal and viscoelastic properties of starches separated from Indica rice Cultivars. Starch/Stärke, 59 , pp. $10-20$

Singh J., Kaur L. \& McCarthy O.J. (2007b). Factors influencing the physic-chemical, morphological, thermal and rheological properties of some chemically modified starches for food applications- A review. Food Hydrocolloids 21, pp. 1-22

Stanley D.W., Stone A.P. \& Tung M.A. (1996). Mechanical properties of food. In Handbook of Food Analysis, Volume I, Ch.4. L.M.L. Nollet (ed.), New York: Marcel Dekker, Inc., 93136

Techawipharat J., Suphantharika M., BeMiller J. N. (2008). Effects of cellulose derivatives and carrageenans on the pasting, paste, andgel properties ofrice starches. Carbohydrate Polymers 73, pp. 417-426

Tsai, M. L., Li, C. F., \& Lii, C. Y. (1997). Effects of granular structure on the pasting behavior of starches. Cereal Chemistry, 74, 750-757. 
Wang Y., Wang L.-J., Li D., Ozkan N., Chen X. D. \& Mao Z.-H. (2008). Effect of flaxseed gum addition on rheological properties of native maize starch. Journal of Food Engineering 89 , pp. $87-92$

Whistler R.L. \& BeMiller J. N. (1997). Carbohydrate Chemistry for Food Scientists. St. Paul, MN: Eagan Press ,pp. 117-164 